\title{
Electromagnetic properties of baryon resonances
}

\author{
Lothar Tiator \\ Institut für Kernphysik, Johannes Gutenberg-Universität, D-55099 Mainz, Germany
}

\begin{abstract}
Longitudinal and transverse transition form factors for most of the four-star nucleon resonances have been obtained from high-quality cross section data and polarization observables measured at MAMI, ELSA, BATES, GRAAL and CEBAF. As an application, we further show how the transition form factors can be used to obtain empirical transverse charge densities. Contour plots of the thus derived densities are shown and compared for the Roper and $S_{11}$ nucleon resonances.
\end{abstract}

Keywords: Baryon resonances, transition form factors

PACS: 14.20.Gk, 13.75.Gx, 13.60.Le

\section{INTRODUCTION}

During the last decade, significant progress on the electromagnetic excitation of nucleon resonances has been obtained. For pion and eta photoproduction very precise data of unpolarized cross sections and photon asymmetries were measured at MAMI@Mainz, ELSA@Bonn, LEGS@Brookhaven and GRAAL@Grenoble. For electroproduction, at Mainz, Bonn and Bates measurements for the $\Delta(1232)$ excitation were performed at low $Q^{2}$, and for higher $Q^{2}$ up to about $6 \mathrm{GeV}^{2}$, at JLab a very large amount of data was collected in an energy range up to the third nucleon resonance region.

In parallel with the ongoing experiments, several theoretical groups developed models and analysis techniques, which were applied to the data. The model-independent GWU/SAID analysis [1] mostly analyzed the pion photoproduction data and improved the values of the photon couplings over the years. Coupled channels analyses were performed by the Giessen group [2] and by the Bonn-Gatchina group [3]. Transition amplitudes were also determined in the framework of dynamically generated resonances by coupling to meson-baryon channels by the Jülich [4] and EBAC [5] groups.

However, most successful concerning the general applicability to the higher resonances, were the unitary isobar models of the Mainz group (MAID model) $[6,7,8]$ and of the JLab group [9] who used dispersion relations as an additional constraint to reduce the model dependence due to incomplete experimental input.

With our unitary isobar model MAID, we analyzed all available electroproduction data in order to determine the transition form factors for all four-star resonances below $W=1.8 \mathrm{GeV}$. In most cases we could obtain both single- $Q^{2}$ and $Q^{2}$ dependent transition form factors for the proton target. In the case of the neutron, the parametrization of the $Q^{2}$ dependence had to take a simpler form because of the much smaller world database.

Single- $Q^{2}$ data points for longitudinal and transverse form factors have been obtained for transitions from the proton to the $\Delta(1232), P_{11}(1440), S_{11}(1535), D_{13}(1520)$, $S_{31}(1620), S_{11}(1650), D_{15}(1675), F_{15}(1680), D_{33}(1700)$ and $P_{13}(1720)$, which can be downloaded from the MAID website [10]. Here only results for the $\Delta(1232), P_{11}(1440)$, 
and the $S_{11}(1535)$ are shown. Full results and details of the parameterizations are given in our recent review article, Ref. [8].

The main motivation for exploring the nucleon transition form factors is to obtain a precise knowledge of the nucleon excitation spectrum, which provides - together with the elastic form factors - a complete description of the nucleon's electromagnetic structure. This structure can be compared with QCD inspired quark models and, in recent years, more and more also with lattice QCD calculations.

\section{First resonance region}

The $\Delta(1232)$ is the only nucleon resonance with a well-defined Breit-Wigner resonance position, $M_{R}=1232 \mathrm{MeV}$. It is an ideal single-channel resonance, the Watson theorem applies, and the Breit-Wigner position coincides with the K-matrix pole position. For these reasons, the $N \rightarrow \Delta(1232)$ form factors can be determined in an essentially model independent way. The magnetic form factor shown in Fig. 1 is very well known already from inelastic electron scattering up to high momentum transfer, $Q^{2}=10 \mathrm{GeV}^{2}$, and can be parameterized in a surprisingly simple form found in our previous MAID analysis,

$$
G_{M}^{*}\left(Q^{2}\right)=3 G_{D}\left(Q^{2}\right) e^{-0.21 Q^{2} / \mathrm{GeV}^{2}}
$$

with $G_{D}$ the standard dipole form factor of the nucleon.

The electric and Coulomb form factors are much smaller and are usually given as ratios of $G_{E}^{*}$ and $G_{M}^{*}$ to the magnetic form factor in percent. Figure 1 also compares the MAID2007 solutions (solid lines) for the ratios $R_{E M}$ and $R_{S M}$ with other analyses. The ratio $R_{E M}$ from MAID2007 stays always below the zero line, in agreement with the original analysis of the data $[11,12]$ and also with the dynamical model of Sato and Lee [13] who concluded that $R_{E M}$ remains negative and tends towards more negative values with increasing $Q^{2}$ instead of an uprise towards unity. This indicates that the predicted helicity conservation at the quark level is irrelevant for the present experimental $Q^{2}$ range. For the ratio $R_{S M}$ our $Q^{2}$ dependent fit approaches a negative constant for large $Q^{2}$ in good agreement with the predictions of Ji et al. [14], Buchmann [15] and Ramalho et al. [16], who use a relation between the ratio $R_{S M}$ and the ratio of the electric and magnetic neutron form factors. For $Q^{2}>1 \mathrm{GeV}^{2}$ our Maid2007 single- $Q^{2}$ analysis for $R_{S M}$ disagrees with the JLab analysis of Aznauryan et al. [18]. Whereas our analysis stays almost constant, the JLab analysis suggests a much larger negative slope. By repeating our data analysis at $Q^{2}=5$ and $6 \mathrm{GeV}^{2}$ in different energy ranges, we found a strong dependence of the fit on the energy interval used. Our results of 2007 were obtained with the full energy range of the measured data, $W=1110-1390 \mathrm{MeV}$.

Figure 1 also shows an analysis (blue open circles) in the energy range of $W=$ $1200-1260 \mathrm{MeV}$, much closer to the resonance position. If we choose the energy interval even closer to resonance, $W=1220-1240 \mathrm{MeV}$, the errors increase further by a factor of 2 and the $E / M$ ratio becomes large and positive, while the $S / M$ ratio remains the same. We conclude that the analysis in this $Q^{2}$ range strongly depends on the energy interval and the parametrization of the background used in the analysis and requires further studies. 

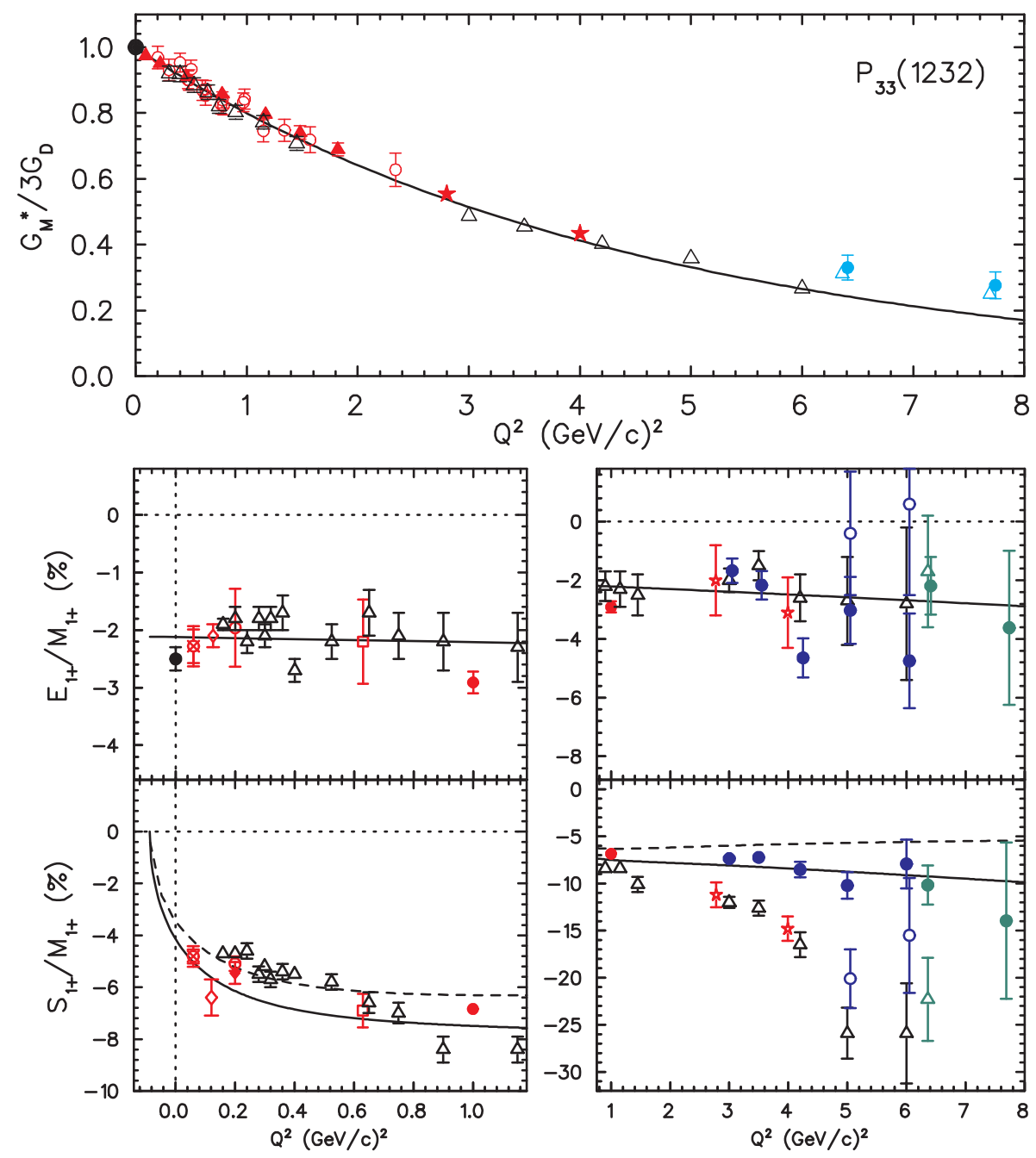

FIGURE 1. Electromagnetic form factors of the $N$ to $\Delta(1232)$ transition. The upper panel shows the magnetic form factor $G_{M}^{*}$ in the definition of Ash and the middle and lower panels show the $E / M$ and $S / M$ ratios, respectively. The curves are the result of our MAID2007 fit to the full dataset of pion electroproduction. The data points are obtained in analyses at fixed $Q^{2}$. The open symbols show the results of the JLab analysis [9], all others are obtained in our MAID analysis. The two largest $Q^{2}$ values $(6.4,7.8)$ show the MAID and the JLab analyses of the cross sections by Villano et al. [17]. For further details, see our review, Ref. [8].

\section{Results for the second resonance region}

The helicity amplitudes for the Roper resonance $P_{11}(1440)$ are displayed in the upper panel of Fig. 2. Our latest $Q^{2}$ dependent solution (solid lines) is in reasonable agreement with the single- $Q^{2}$ analysis (red circles). The figure shows a zero crossing of the transverse helicity amplitude $A_{1 / 2}\left(Q^{2}\right)$ at $Q^{2} \approx 0.7 \mathrm{GeV}^{2}$ and a maximum at the relatively large momentum transfer $Q^{2} \approx 2.0 \mathrm{GeV}^{2}$. The longitudinal Roper excitation $S_{1 / 2}\left(Q^{2}\right)$ rises to large values around $Q^{2} \approx 0.5 \mathrm{GeV}^{2}$ and produces one of the strongest 

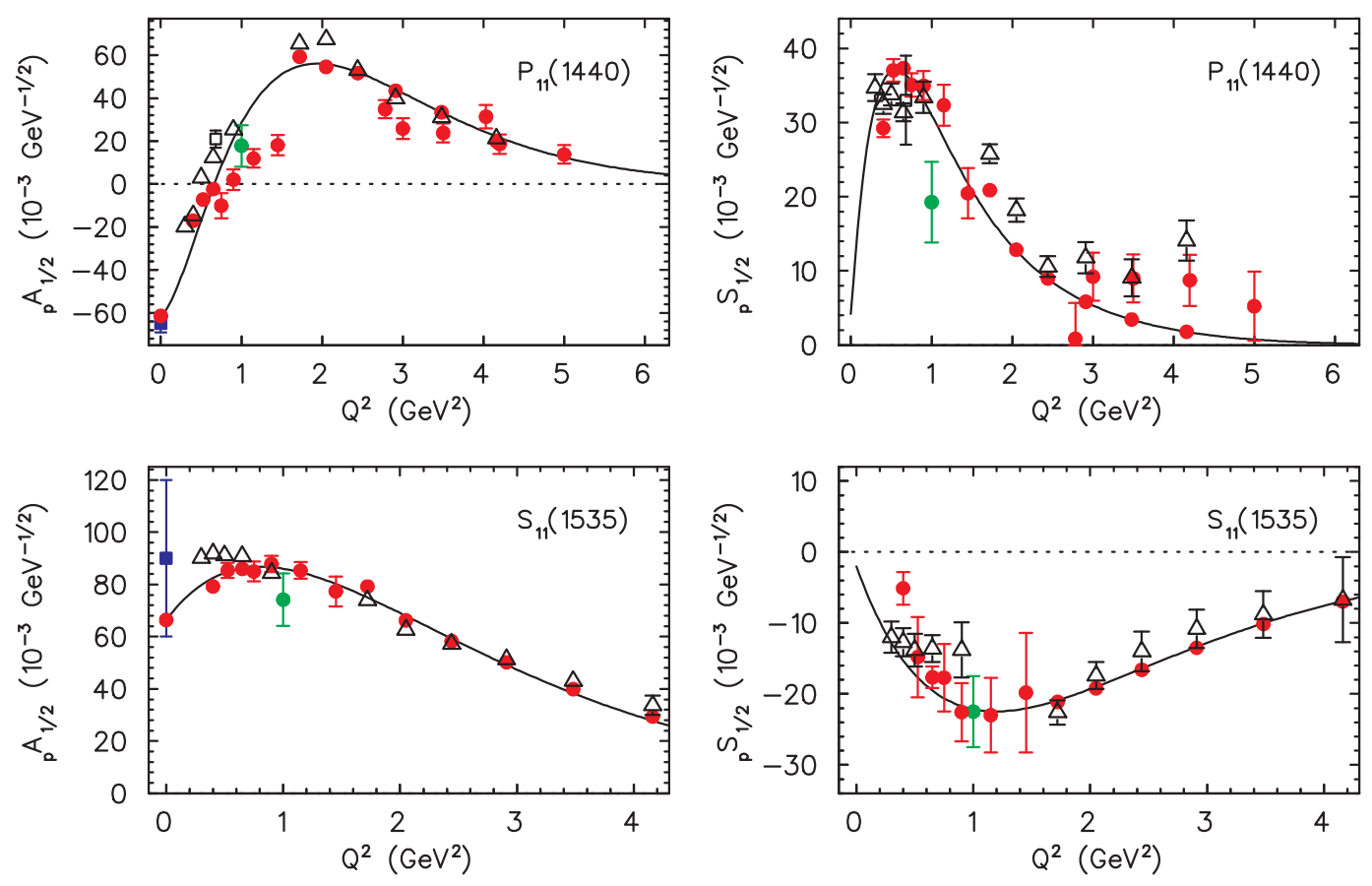

FIGURE 2. Transverse $\left(A_{1 / 2}\right)$ and longitudinal $\left(S_{1 / 2}\right)$ electromagnetic form factors of the $p$ to $P_{11}(1440)$ (upper panel) and $p$ to $S_{11}(1535)$ (lower panel) transitions. The curves are the result of our MAID2007 fit to the full dataset of pion electroproduction. The data points are obtained in analyses at fixed $Q^{2}$. The open symbols show the results of the JLab analysis $[18,17]$, all others are obtained in our MAID analysis. For further details, see Ref. [8].

longitudinal amplitudes that we find in our analyses.

The lower panel in Fig. 2 displays the results for the $S_{11}(1535)$ resonance. The red single- $Q^{2}$ data points show our results of 2007, which are in good agreement with our $Q^{2}$ dependent analysis (solid lines). The black triangles are the 2009 results of Ref. [18]. The blue data point at $Q^{2}=0$ represents the PDG average over several $\gamma, \pi$ and $\gamma, \eta$ analyses. While we find values around 65 in all MAID analyses, the JLab analysis obtains values around 90 for $\gamma, \pi$ and 110 for $\gamma, \eta$ [9]. Also the SAID and Bonn-Gatchina groups extract values around 100, but in a very recent analysis, based on pion photoproduction, Shrestha and Manley report, however, also a small coupling of 59(3) [19].

From the $Q^{2}$ dependent parametrization of the transition form factors we can calculate transverse charge transition densities, as viewed from a light front moving towards the baryon [20]. For that we first have to transform the helicity form factors $A_{1 / 2}, S_{1 / 2}$ to the Dirac-like form factors $F_{1}^{N N^{*}}$ and $F_{2}^{N N^{*}}$, which results in simple linear relations. The transverse densities are then obtained by a 2-dim Fourier-Bessel transformation. The densities relating to $F_{1}$ appear as fully symmetrical monopole patterns, while the $F_{2}$ form factors transform to additional dipole patterns (further details in Ref. [8]).

As an example, in Fig. 3 we show the polarized quark transverse charge densities from the proton to the Roper and to the $S_{11}(1535)$ resonances. Comparing these results, we find that the dipole contribution of the up and down quarks to the polarized densities is much less pronounced for the $S_{11}$ due to the much smaller $F_{2}^{N N^{*}} / F_{1}^{N N^{*}}$ form factor ratio. 

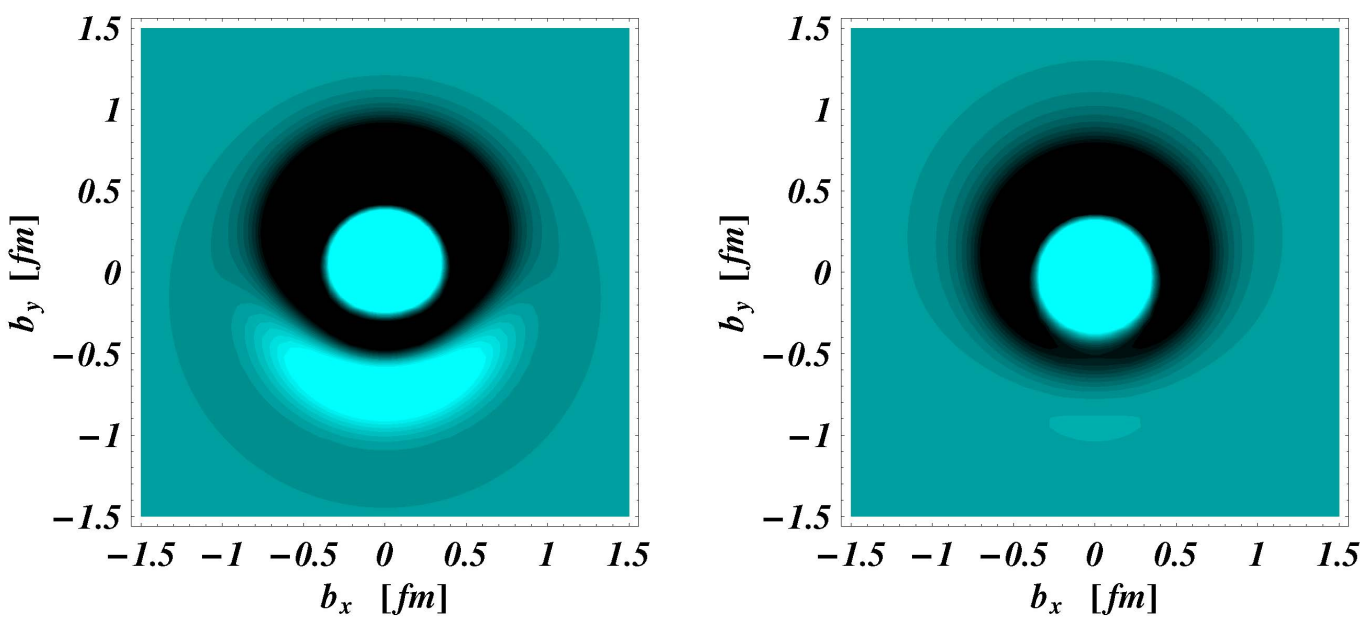

FIGURE 3. Comparison of the polarized quark transverse transition charge densities corresponding to the e.m. transitions $p \rightarrow P_{11}(1440)\left(\frac{1}{2}^{+} \rightarrow \frac{1}{2}^{+}\right)$(left panel) and $p \rightarrow S_{11}(1535)\left(\frac{1}{2}^{+} \rightarrow \frac{1}{2}^{-}\right)$(right panel). The light (dark) regions correspond to positive up quark (negative down quark) densities.

\section{ACKNOWLEDGMENTS}

We want to thank the Deutsche Forschungsgemeinschaft for the support by the Collaborative Research Center 1044.

\section{REFERENCES}

1. R. L. Workman, W. J. Briscoe, M. W. Paris and I. I. Strakovsky, Phys. Rev. C 85, 025201 (2012). http: / / gwdac.phys.gwu.edu

2. V. Shklyar, H. Lenske and U. Mosel, Phys. Lett. B650, 172 (2007).

3. A. V. Anisovich et al., Eur. Phys. J. A44, 203 (2010).

http://pwa.hiskp.uni-bonn.de

4. M. Döring and K. Nakayama, Eur. Phys. J. A43, 83 (2010).

5. N. Suzuki, T. Sato and T. -S. H. Lee, Phys. Rev. C 82, 045206 (2010).

6. D. Drechsel, O. Hanstein, S. S. Kamalov and L. Tiator, Nucl. Phys. A 645, 145 (1999).

7. D. Drechsel, S. S. Kamalov and L. Tiator, Eur. Phys. J. A 34, 69 (2007).

8. L. Tiator, D. Drechsel, S. S. Kamalov and M. Vanderhaeghen, Eur. Phys. J. ST 198, 141 (2011).

9. I. G. Aznauryan and V. D. Burkert, Prog. Part. Nucl. Phys. 67, 1 (2012).

10. MAID website: http://kph.uni-mainz.de/MAID/, amplitude data: http: / / kph . uni-mainz.de/MAID/maid2007/data.html

11. M. Ungaro et al. [ CLAS Collaboration ], Phys. Rev. Lett. 97, 112003 (2006).

12. V. V. Frolov et al., Phys. Rev. Lett. 82, 45 (1999).

13. T. Sato and T.-S. H. Lee, Phys. Rev. C 63, 055201 (2001).

14. X.-d. Ji, J.-P. Ma and F. Yuan, Phys. Rev. Lett. 90, 241601 (2003).

15. A. J. Buchmann, Phys. Rev. Lett. 93, 212301 (2004).

16. G. Ramalho, M. T. Pena and F. Gross, Phys. Rev. D 78, 114017 (2008).

17. A. N. Villano et al., Phys. Rev. C80, 035203 (2009).

18. I. G. Aznauryan et al. [ CLAS Collaboration ], Phys. Rev. C80, 055203 (2009).

19. M. Shrestha and D. M. Manley, arXiv:1208.2710 [hep-ph].

20. L. Tiator and M. Vanderhaeghen, Phys. Lett. B 642, 344 (2009). 